\title{
Diabetics at School Assessment of Knowledge and Attitudes of School Staff
}

\author{
Roberta Pujia, $M D^{1}$, Filippo Dell'Apa, $M D^{2}$, Mimma Caloiero, $M D^{3}$, Domenico Sinopoli, $N B^{4}$, Stefania \\ Zampogna, $M D^{4}$, Rosa Anfosso, $M D^{5}$ and Antonella Centonze, $M D^{4}$
}

${ }^{1}$ Department of Medical and Surgical Sciences, Magna Graecia University Catanzaro, Italy

${ }^{2}$ Department of Women's and Children's Health - University of Padua, Italy

${ }^{3}$ Pediatric Unit, Lamezia Terme Hospital, Italy

${ }^{4}$ Azienda Ospedaliera Pugliese- Ciaccio - Catanzaro, Italy

${ }^{5}$ Head of the health promotion service - ASP Catanzaro, Italy

\begin{abstract}
Type 1 diabetes mellitus is the most frequent endocrinopathy of childhood. Epidemiological data have indicated that its incidence is constantly increasing, especially in the age group of fewer than 5-years. Social and health policies must take account of these new problems. The school, as an educational agency, is a central part of the child's life, being the place where he or she spends most of the day in a continuous interaction with classmates and teachers. It has a decisive impact on they growth and they social integration process. The teacher must also learn all the practical skills and abilities necessary, manage any hyper-hypoglycemic crises and emergencies, without drawing attention to any perceived differences between the children.
\end{abstract}

The aim of the study is to identify the teachers' knowledge of the pathology and demonstrate how the training of school staff can as a priority guarantee the safety, as well as the educational integration of the child with diabetes.

\section{Keywords}

Type 1 diabetes, School, Children training, Administration drugs, Protocols, Emergencies

\section{Introduction}

Type 1 diabetes mellitus is the most frequent endocrinopathy of childhood. Epidemiological data indicate that its incidence is constantly increasing, especially in the under five years age group. Social and health policies must take into account these new problems that equally require new solutions within the population. In particular, as regards the school it is necessary that attendance at school takes place in conditions of security and calm for the diabetic student, whose condition can lead to feelings of being different and marginalization, and equally of security and confidence for teachers, called to act in the daily management of the pathology in order to guarantee the right to study, the continuing good health and the full integration of the pupil, allowing they to participate in all school activities [1,2].

The school, as an educational agency, constitutes a central feature in the life of the child, being the place where he spends most of the day in continuous interaction with classmates and teachers. The school is also the first important step in integration and socialization of the child, the place where he builds his personality, recognizes his individuality in the community and sense of belonging. The integration at school of the child with diabetes is sensitive and crucial because it has a decisive impact on his growth and on his social integration. The child at school has to deal with some aspects related to his diabetic condition, such as blood glucose control, insulin intake, sports practice, diet, but also the teacher must acquire technical skills and practical skills, in so as to be able to adequately manage any hyper-hypoglycemic crises and emergencies, without forgetting the role of breaking down any barrier that can make people perceive any elements of being different. All the participants involved in the problem must be secure: The pupil must live in a con-

*Corresponding author: Centonze Antonella, MD, Azienda Ospedaliera Pugliese-Ciaccio Hospital, Via Pio X, Catanzaro, Italy, Tel: 3667242376

Accepted: April 18, 2020

Published online: April 20, 2020

Citation: Pujia R, Dell'Apa F, Caloiero M, et al. (2020) Diabetics at School Assessment of Knowledge and Attitudes of School Staff. Clin Pediatr Res 4(1):72-76 
dition of normality and calm, without the pathology having negative repercussions on his self-image, teachers must be trained in such a way as not to be anxious about the management of the diabetic child and parents must develop a coordinated and joint action with the teaching staff.

The aim of our work was to assess the state of knowledge of the pathology by teachers and demonstrate how structured and compulsory education for school staff can certainly guarantee the safety, as well as the educational integration of the child with diabetes.

\section{Materials and Methods}

In 2017 the health promotion department of the Italian province of Catanzaro carried out training courses aimed at teachers of primary and secondary schools. The course "A Diabetes School" was carried out in 11 schools in the province of Catanzaro. 156 teachers participated in the training event. Before the information/training course a "pre-test" questionnaire was given to the teachers; the same "post-test" questionnaire was given at the end of the course.

\section{Results and Discussion}

11 schools in the province of Catanzaro and 156 teachers were involved. We subjected the teachers to a pre-test questionnaire before the training course and a post-test questionnaire.

\section{Pre-test considerations}

“Has a diabetes course been done at your school?"

$100 \%$ of teachers answered no.

"Do you think you have sufficient knowledge about diabetes?"

$14 \%$ of the teachers answered yes, $86 \%$ answered no.

"Do you find it difficult to manage a diabetic pupil?"

$36 \%$ of teachers answered yes, $64 \%$ answered no.

\section{Post-test consideration}

"Do you know the symptoms of hypoglycemia?"

Pre-test: $83 \%$ answered no - $17 \%$ answered yes.

Post-test: 4\% answered no, 96\% answered yes.

"Do you know about hypoglycemia therapy?"

Pre-test: No: $92 \%$, yes: $8 \%$.

Post-test: $4 \%$ no, $96 \%$ yes.

"Do you know the values of hypoglycemia?"

Pre-test: $94 \%$ no, 6\% yes.

Post-test: $7 \%$ no, $93 \%$ yes.

"Do you know the symptoms of hyperglycemia?"

Pre-test: $88 \%$ no, $12 \%$ yes.

Post-test: $11 \%$ no, $89 \%$ yes.

"Do you know about hyperglycemia?"

Pre-test: $90 \%$ no, $10 \%$ yes.
Post-test: $9 \%$ no, $91 \%$ yes.

"Which of these situations is an emergency?"

Pre-test: Hyperglycemia 63\%, hypoglycemia 37\%.

Post-test: hyperglycemia $10 \%$, hypoglycemia $90 \%$.

"Indicate one or more foods with simple sugars".

Pre-test: $48 \%$ correct answers, 52\% wrong answers.

Post-test: $1 \%$ incorrect, $99 \%$ accurate.

"Indicate one or more foods with compound sugars".

Pre-test: 33\% correct answers, 67\% wrong answers.

Post-test: 99\% correct answers, 1\% incorrect.

"Do you think the diabetic child can do sports at school?"

Pre-test: $59 \%$ no, $41 \%$ yes.

Post-test: $91 \%$ yes, $9 \%$ no.

"Do you know the precautionary measures in case of having a party in the classroom?"

Pre-test: $89 \%$ no, $11 \%$ yes.

Post-test: $91 \%$ yes, $9 \%$ no.

"Glucagon is administered in the event of".

Pre-test for: $76 \%$, ipo $24 \%$.

Post-test: 95\% hypoglycemia, 5\% hyperglycemia.

"Glucagon is administered":

Pre-test: Intramuscular 26\%, 62\% non-responsive, other (sublingual, drip, os) $12 \%$.

Post- test: $100 \%$ intramuscular.

"The course met its expectation?"

$74 \%$ absolutely, $26 \%$ adequate, pointless.

"Did the course allow you to acquire knowledge, skills and competences?"

Yes $100 \%$.

"The topics covered are":

Very interesting: $87 \%$, quite interesting: $13 \%$, uninteresting: $0 \%$, not at all interesting: $0 \%$.

"What do you think was the most interesting topic of the course?"

Hypoglycemia $13 \%$, all $47 \%$, therapy $6 \%$, Glucagon $7 \%$, emergency management $9 \%$, child can do everything $1 \%$, does not respond 17\%, 4\% symptoms Table 1.

\section{Conclusion}

Type 1 diabetes (T1D) is one of the most common endocrine and metabolic conditions in childhood, showing an increasing incidence rate of about 3\% per year during the last two decades [3]. During the period 1990-2003 the incidence rate in Italy was 12.26 per 100,000 person years, with an increasing temporal trend of $2.94 \%$ per year. 
Citation: Pujia R, Dell'Apa F, Caloiero M, et al. (2020) Diabetics at School Assessment of Knowledge and Attitudes of School Staff. Clin Pediatr $\operatorname{Res} 4(1): 72-76$

Table 1: The table summarize in percentage the answers to the questions submitted to teachers both in the pre-test and in the post-test.

\begin{tabular}{|c|c|c|c|c|c|c|}
\hline & \multicolumn{3}{|l|}{ Pretest } & \multicolumn{3}{|l|}{ Post test } \\
\hline & \multicolumn{2}{|l|}{ no } & yes & no & \multicolumn{2}{|c|}{ yes } \\
\hline Do you know the symptoms of hypoglycemia? & \multicolumn{2}{|l|}{$83 \%$} & $17 \%$ & $4 \%$ & \multicolumn{2}{|c|}{$96 \%$} \\
\hline Do you know hypoglycemia therapy & \multicolumn{2}{|l|}{$92 \%$} & $8 \%$ & $4 \%$ & \multicolumn{2}{|c|}{$96 \%$} \\
\hline Do you know the values of hypoglycemia? & \multicolumn{2}{|l|}{$94 \%$} & $6 \%$ & $7 \%$ & \multicolumn{2}{|c|}{$93 \%$} \\
\hline Do you know the symptoms of hyperglycemia? & \multicolumn{2}{|l|}{$88 \%$} & $12 \%$ & $11 \%$ & \multicolumn{2}{|c|}{$89 \%$} \\
\hline Do you know about hyperglycemia? & \multicolumn{2}{|l|}{$90 \%$} & $10 \%$ & $9 \%$ & \multicolumn{2}{|c|}{$91 \%$} \\
\hline Do you believe that the diabetic child can do sport at school? & \multicolumn{2}{|l|}{$59 \%$} & $41 \%$ & $91 \%$ & \multicolumn{2}{|c|}{$9 \%$} \\
\hline \multirow{2}{*}{$\begin{array}{l}\text { Do you know the precautionary measures to take in case of } \\
\text { a party in the classroom? }\end{array}$} & \multicolumn{2}{|l|}{$89 \%$} & $11 \%$ & $91 \%$ & \multicolumn{2}{|c|}{$9 \%$} \\
\hline & \multicolumn{2}{|l|}{ Hyperglycemia } & Hypoglycemia & Hyperglycemia & \multicolumn{2}{|c|}{ Hypoglycemia } \\
\hline Which of these situations is an emergency? & \multicolumn{2}{|l|}{$63 \%$} & $37 \%$ & $10 \%$ & \multicolumn{2}{|c|}{$90 \%$} \\
\hline Glucagon is administered in the event of: & $76 \%$ & \multicolumn{2}{|c|}{$24 \%$} & $5 \%$ & \multicolumn{2}{|c|}{$95 \%$} \\
\hline Glucagon is administered? & Pre-test & & & Post -test & & \\
\hline & Intramuscularly & other & not answer & intramuscularly & other & $\begin{array}{l}\text { not } \\
\text { answer }\end{array}$ \\
\hline & $26 \%$ & $12 \%$ & $62 \%$ & $100 \%$ & $0 \%$ & $0 \%$ \\
\hline
\end{tabular}

The most recent snapshot of the diabetes situation in Italian schools is the one made in the Dawn Youth Project in 2008 , with the Alba project (longitudinal analysis of the needs of adolescents with diabetes) [4-6].

The aim of the project was to detect the ways in which diabetes is managed during school hours (self-checks, insulin administration, physical activity, etc.) in primary and secondary schools, and to assess the most frequent concerns and discomforts that children, families and teachers are faced with, highlighting any barriers (social and personal) that can contribute to creating discrimination.

Key data emerged which highlighted:

- Lack of a shared protocol outlining how to accommodate a child with diabetes at school.

- Wide spread, poor knowledge of the pathology that sometimes creates an inflexible attitude and prejudices in the school staff.

- Difficulty in ensuring the administration of insulin.

- Lack of trained personnel within the school who can handle any emergencies.

- Lack of structured and compulsory training for school staff who have a child with diabetes in the institution.

- Difficulty for pediatric diabetology centers to organize training courses on a continuous basis, as they often do not have sufficient human resources to meet such a demand.

In November 2005, the Italian Ministry of Education and Ministry of Health issued recommendations for the administration of drugs during school hours, taking into account the possibility of their administration by non-medical personnel, provided that this practice did not require specialist knowledge, did not call for discretion and was on a voluntary basis, while referring the management of emergencies, in a rather ambiguous way, to the National Health Service. These recommendations have had the merit of raising the issue, but certainly not of facing it, precisely because of their exhortative nature.

However, even where the regional protocols are in place, the problem of the management of emergencies and in particular the administration of glucagon has not been clearly and unambiguously resolved.

Some considerations can help us to understand some crucial aspects. It is by now an established precedent that enrollment at school creates a legal duty of protection and vigilance of a contractual nature; the protection of the right to health in schools comes into this scenario and the head teacher must plan and implement all the measures necessary to guarantee it.

From the obligation to watch over the child, in order to stop him injuring himself, follows the duty to prevent situations of risk arising from the lack of the routine administration of drugs and, more particularly, from the failure to administer glucagon in an emergency $[2,7]$.

It seems fundamental that the prevention of severe hypoglycemia, would be delivered through the training of school staff who are aware of the factors that can generate a hypoglycemic episode, who can recognize the first signs that accompany it and who know how to take the appropriate corrective measures.

On the other hand, if insulin administration is something hat school staff can only do with spontaneously, child protection and therefore the prevention of hypoglycemia or the treatment of mild hypoglycemia, are part of the good practice that the responsible practitioner must have in the same as a responsible parent. 
We know that the educationalists' knowledge of the disease is fundamental to lower the barriers and fears, which can lead to either the rejection or excessive protection of the child with diabetes, and that training is the tool to make the school environment cozy and safe for the child with diabetes. The strategy document shows that the School plays a dual role: First of all it represents an important point in the gradual process of acceptance of diabetes and autonomy on the part of the child; secondly, it constitutes an opportunity for food education for classmates and a cascade effect for families.

The dissemination of this document and its adoption as a technical tool by the Ministries of Health and Education to develop national guidelines that go beyond merely regional grouping and assume a mandatory nature, would allow children/young people and their families to perceive the school as a safe, welcoming and inclusive place [1-3,8,9].

The estimated prevalence of type 1 diabetes is about $0.5 \%$ of the general population, with incidence values varying from region to region, on average about 10-12 cases/ year/100,000 inhabitants, as reported in the National Diabetes Plan. Furthermore, it is stated that, in recent years, the age of onset of the disease has dropped.

In the 2016 National Chronicity Plan, whose aim is to contribute to the improvement of the protection for people suffering from chronic diseases, reducing the burden on the individual, their family and the social context, improving the quality of life, making health services more effective and efficient in terms of prevention and assistance and ensuring greater uniformity and fairness of access to citizens, the school appears as the main place in which to promote, as far as children with chronic diseases are concerned, a relative growth similar to their peers. The Plan affirms the necessity to carry out campaigns to communicate and inform, as well as to prepare suitable training routes aimed at families and schools, with the support of relevant associations and scientific societies, to support the culture of chronic illness in the pediatric age and ensure the safety of children in school, through the administration of the necessary medicines according to the ministerial guidelines.

The principle underlying the model of school intervention is the demedicalization of students with chronic diseases, in particular diabetes, asthma and epilepsy, which is seen to be the absence of health professionals of any kind within the school.

This process involves the training of school staff, who are acentral figure in the reception process, in the management of continuity of therapy and possible emergencies.

Parents know that the unwillingness of the school is not due to a lack of will, but rather the lack of adequate training (probably those completed so far have not been sufficient to develop confidence and a sense of empowerment in the school community) and the presence of national guideline that protect both the children and the family, as well as the school staff, reinforcing the sense of solidarity that is vital for putting oneself voluntarily "at stake".
This is why monitoring projects that verify the effectiveness of the model in the field are desirable: The results would be extremely useful in finalizing the model itself. In this regard, we are working on the "School and Diabetes" Project, which aims to analyze the feasibility and sustainability of the model in the field $[2,10]$.

Children spend a lot of time at school; in the case of a child with diabetes it is essential that school attendance take place in order to ensure well-being and safety. This is why it is important that the school staff know the needs of a child with diabetes and the basic principles of his treatment, so as to guarantee the right to education and health as well as his full integration.

Even though the law 115 of 1987 stated that "the regional interventions are aimed at facilitating the inclusion of diabetics in school, sports and work activities", in 2008 the Alba project (longitudinal analysis of the needs of adolescents with diabetes) highlighted several criticisms such as poor knowledge of the disease by school staff, difficulty in insulin administration, lack of a management plan for possible emergencies, lack of structured and compulsory training for school staff, difficulties on the part of pediatric diabetology to organize ongoing training courses due to lack of staff $[1,2]$.

The results reveal an inadequate knowledge of juvenile diabetes, attested by the pre-test on diabetes and its treatment, a situation that could have dangerous consequences for the health and life of the child and affect his physical psycho-growth and self-esteem.

Most of the school staff lacked adequate information on diabetes and the interventions to be carried out if necessary. Contact between parents and school is not always perfect and therefore not sufficient to fill gaps in the teachers' knowledge and the information provided by the hospital is rarely adequate.

Since promoting health is not merely giving assistance, but the involvement of all the competent and responsible people at various levels, and promoting the education and educational success of each child is the task and responsibility of the school, the need emerges for total synergy between the players in education and health care to provide information/training and cascade welfare information down through the school population. It is recommended to better inform the school staff on the subject, as well as implement early and repeated contacts between hospital and school.

\section{References}

1. Ludvigsson J (1997) Diabetics in School. Knowledge and attitudes of school staff in relation to juvenile diabetics. Scand J Soc Med 5: 21-30.

2. R Olympia RP, Wan E, Avner JR (2005) The preparedness of school to respond to emergencies in children: A national survey of school nurses. Pediatrics 116: e738-e745.

3. Patera IP, La Loggia $A$ (2014) Attività diabetologica e metabolica in italia-diabete e scuola. G It Diabetol 34: 163-168.

4. Ministro dell'Istruzione dell'Universita e della Ricerca-Ministro della Salute (2013) Raccomandazioni sulla somministrazione dei 

Res 4(1):72-76

farmaci in orario scolastico (nota prot. 2312 Dip/Segr) 25 novembre 2005. Piano sulla malattia diabetica-GURI 32:9.

5. https://www.agditalia.it/scuola-e-diabete/documento-strategico-e-le-norme

6. http://www.ibdo.it/pdf/07_Diabete-e-scuola.pdf

7. Bruno G, Maule M, Merletti F, et al. (2010) Age-period cohort analysis of 1990-2003 incidence time trends of childhood diabetes in Italy. The RIDI Study. Diabetes 59: 2281-2287.
8. (2014) Diabetes Care in the School and Day Care Setting Diabetes Care. 37.

9. Chiara Giorgetti, Lucia Ferrito, F Zallocco, et al. (2016) Organization and regional distribution of centers for the management of children and adolescents with diabetes in Italy. Ital J Pediatr 42: 33.

10. Dettaglio atto (1987) Disposizioni per la prevenzione e la cura del diabete mellito. Legge 16 marzo 115. 